\title{
Andrew M. Evens and Kristie A. Blum (Eds), Non-Hodgkin Lymphoma. Pathology, Imaging, and Current Therapy
}

\author{
Springer International Publishing Switzerland 2015, ISBN: 978-3-319-13149-8
}

\author{
Sara Pellegrino ${ }^{1} \cdot$ Luigi Mansi $^{1}$
}

Published online: 23 March 2016

(C) Springer-Verlag Berlin Heidelberg 2016

This book represents the 165 th volume in the series Cancer Treatment and Research, edited by renowned oncologist Steven T. Rosen, who also serves as Provost and Scientific Director of the City of Hope in Duarte, California. The editors of this volume are two internationally recognized experts in hematology/oncology: Andrew M. Evens, Director of the Tufts Cancer Center in Boston, and Kristie A. Blum, Professor of Internal Medicine at Ohio State University in Columbus.

The book comprises 344 pages, divided into 14 chapters and enriched by an impressive bibliography. The theme of this volume is non-Hodgkin's lymphoma, and it provides a wide range of details regarding epidemiology, pathogenesis, imaging and therapy, including new treatment frontiers involving the use of experimental antibodies and proteasome inhibitors in addition to stem cell transplantation.

The text features a discussion of each characteristic feature of non-Hodgkin's lymphoma, from the various diagnostic and therapeutic approaches for each histologic subtype, to the distinctive etiology, imaging, and gene expression profiling of the disease.
In this regard, the fourth and fifth chapters deserve separate mention. The fourth chapter is centered on the different gene expression profiles among the various histotypes of nonHodgkin's lymphoma, which underscores the emerging role of the latter in determining an appropriate therapeutic approach. The fifth chapter provides a detailed description of the effectiveness of PET-CT with FDG in staging and follow-up, the importance of interim PET-CT in the early evaluation of tumor response, and ongoing research in new radiotracers beyond FDG, with broad prospects for the future. Another interesting chapter is the penultimate, which examines post-transplant lymphoproliferative disorders and provides accurate definitions, from causative factors to prognostic indexes, focusing on both current therapeutic strategies and new therapeutic perspectives.

The writing in the book is fluent and easy to read, with text that flows well even when dealing with complex arguments, enriched by explanatory images and tables.

We can highly recommend this book, not only to experts, but to students and to all clinicians who have an interest in non-Hodgkin's lymphoma.
Luigi Mansi

luigi.mansi@unina2.it

1 Second University of Naples, Naples, Italy 